Proc. XIX International School of Semiconducting Compounds, Jaszowiec 1990

\title{
ACCEPTOR-LIKE LEVEL OF THE EL2 DEFECT IN ITS METASTABLE CONFIGURATION*
}

\author{
P. DRESZER, \\ High Pressure Research Center."Unipress”, Polish Academy of Sciences, Sokolowska \\ 29/37, 01-142 Warszawa, Poland \\ M. BAJ AND A. BABIŃSKI \\ Institute of Experimental Physics, Warsaw University, Hoża 69, 00-681 Warszawa, \\ Poland \\ (Received August 8, 1990)
}

\begin{abstract}
This paper presents for the first time the evident experimental confirmation that EL2 defect, while being in its metastable configuration, traps undex hydrostatic pressure an additional electron, i.e. the acceptor-like (EL2 $\left.{ }^{*}\right)^{0 /-}$ level enters the energy gap under pressure. We propose that in $n-G a A s$ the EL2 thermal recovery takes always place via the (EL2*) ${ }^{-}$state.
\end{abstract}

PACS numbers:71.55.Eq, 78.50.Ge

Our recent experimental results showed that under pressure of $p \geq 0.3 \mathrm{GPa}$ the metastable configuration of the EL2 defect in GaAs became optically sensitive and a very efficient pure optical recovery process was observed [1]. We suggested that these facts could be related to the acceptor-like level of the metastable EL2 - $\left(\mathrm{EL} 2^{*}\right)^{0 /-}$, entering the gap under pressure. In this work we present the experimental evidence of the above suggestion. We investigated two $n$-GaAs samples: \#5B with $n_{300 \mathrm{~K}}=1.2 \times 10^{16} \mathrm{~cm}^{-3}, N_{\mathrm{EL} 2}=2.7 \times 10^{16} \mathrm{~cm}^{-3}$ and \#15C with $n_{300 K}=3.2 \times 10^{16} \mathrm{~cm}^{-3}, N_{\mathrm{EL} 2}=1.3 \times 10^{16} \mathrm{~cm}^{-3}$. Clear manifestation of the $\left(\mathrm{EL} 2^{*}\right)^{0 /-}$ level was observed in electrical (conductivity and DLTS) measurements.

At temperatures $T<T_{c}$ (where $T_{c}$ - temperature at which EL2 thermally recovers) and sufficiently high pressure ( $p \geq 0.3 \mathrm{GPa})$ during subsequent optical quenching and optical recovery processes strong persistent changes of the electrical conductivity were observed. This was the consequence of the trapping and releasing of the electrons by EL2 optically transferred between its normal (EL2 ${ }^{\circ}$ ) and

*This work is supported by CPBP 01.05 Program of the Polish Ministry of National Education 

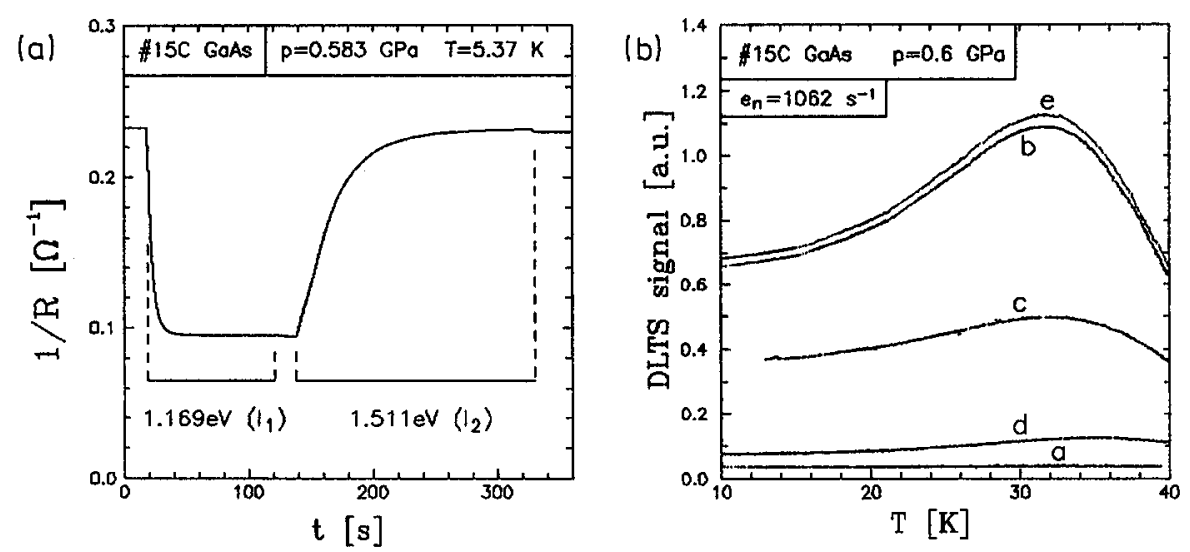

Fig. 1. (a) The electrical conductance of the \#15C sample during the succeeding pure optical EL2-quenching $(1.169 \mathrm{eV})$ and optical EL2-recovery $(1.511 \mathrm{eV})$ processes. The ratio of the monochromatic light intensities was $I_{1}: I_{2}=5: 1$. (b) The influence of the succeeding processes of the monochromatic light irradiation performed at $T=5 \mathrm{~K}$ on the DLTS spectra for \#15C sample: a - initial DLTS spectrum after cooling the sample down in the dark; b - after $1.13 \mathrm{eV}$ EL2-photoquenching for $10 \mathrm{~min}$; c - after $1.57 \mathrm{eV}$ partial EL2-photorecovery for $5 \mathrm{~min}$; $\mathrm{d}$ - the same as c after next $5 \mathrm{~min}$; e - after 1.13 eV EL2-photoquenching for $10 \mathrm{~min}$.

metastable (EL2*) configurations (see Fig. 1a). For the sample \#5B , for which all the free electrons could be trapped by the metastable EL2, these persistent conductivity changes at $T=5 \mathrm{~K}$ and $p=0.65 \mathrm{GPa}$ exceeded 7 orders of magnitude! At $T<T_{c}$ and sufficiently high pressure the electrical conductivity of the samples with metastable EL2 was strongly temperature-dependent which reflected thermal equilibrium between the (EL2*) ${ }^{0 /-}$ level and the conduction band.

At $T<T_{c}$ and high pressure we observed DLTS signal originating from the $\left(\text { EL2 }^{*}\right)^{0 /-}$ level. The DLTS signal intensity was correlated with the concentration of the EL2 in its metastable configuration (see Fig. 1b). Moreover, the position of the DLTS peak shifted to higher temperatures with increasing pressure.

At a constant temperature $T<T_{c}$, while the EL2 was metastable, we observed pressure induced changes of the electrical conductivity originating from the trapping of free electrons by the (EL2*) ${ }^{0 /-}$ level. This trapping is seen in Fig. 2a together with the curves calculated assuming that the energy lewel is equal to $16 \mathrm{meV}$ above the conduction band edge at $p=0$ and the pressure shift is $-68 \mathrm{meV} / \mathrm{GPa}$.

All the above effects, which were attributed to the (EL2* $)^{0 /-}$ level, were not seen without pressure and under pressure they disappeared at temperature $T_{c}$ at which EL2 thermally recovered from its metastable configuration.

We observed very characteristic, nonmonotonous pressure dependence of $T_{c}$ with the minimum close to the pressure at which the $\left(\mathrm{EL} 2^{*}\right)^{0 /-}$ level enters the 

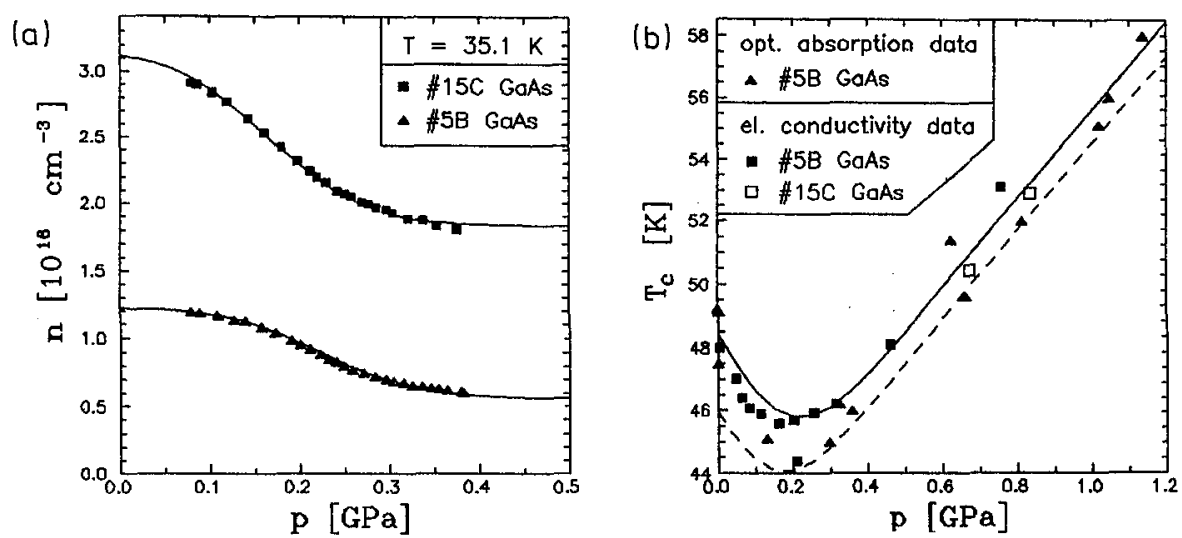

Fig. 2 (a) The hydrostatic pressure dependence of the free electron concentration after full or partial EL2-photoquenching for \#15 C and \#5 B samples, respectively. The solid lines were fitted to experimental data to determine the $\left(\mathrm{EL} 2^{*}\right)^{0 /-}$ level energy and its pressure shift - see text. (b) Pressure dependence of $T_{c}$. The broken and solid lines are the results of calculation performed for \#15 C and \#5 B samples, respectively (see text).

gap (see Fig. 2b). Taking into account the existence of this level we propose that in n-GaAs the EL2 thermal recovery takes place via the (EL2*)- state - i.e. the rate of the thermal recovery is not directly dependent on free electron concentration (like in so-called Auger mechanism $[2,3]$ ) and the rate equation is as follows:

$$
\frac{d N_{\mathrm{EL} 2^{0}}}{d t}=\alpha_{0} \times \exp \left(-E_{B} / k T\right) \times N_{\left(\mathrm{EL} 2^{*}\right)}
$$

where $N_{E L 2^{\circ}}$ is the concentration of the EL2 defect in its neutral charge state and its normal configuration, $N_{\left(E L 2^{*}\right)^{-}}$is the concentration of the negatively charged E L2 defect being in its metastable configuration, $\alpha_{0}$ is the preexponential factor, and $E_{B}$ is the configurational barrier which has to be overcome during the $\left(\mathrm{EL} 2^{*}\right)^{-} \rightarrow \mathrm{EL} 2^{0}$ recovery process.

Assuming thermal equilibrium between the $\left(\mathrm{EL}^{*}\right)^{0 /-}$ level and the conduction band we solved the above rate equation. The best agreement between the experimental and calculated $T_{c}(p)$ dependence were obtained for $E_{B}=75 \mathrm{meV}+$ $p \times 28 \mathrm{meV} / \mathrm{GPa}$. The results of the calculations clearly explain the experimental data (see Fig. 2b). It should be pointed out that within our model we could describe the pressure dependence of $T_{c}$ only if we took into account the appropriate pressure shift of $\left(\mathrm{EL} 2^{*}\right)^{0 /-~ l e v e l . ~}$

\section{Acknowledgments}

The authors are very grateful to Dr. J. Lagowski for the GaAs samples. 


\section{References}

[1.] M. Baj, P. Dreszer, Phys. Rev. B 39, 10470 (1989.)

[2.] G. Vincent, D. Bois, A. Chantre, J. Appl. Phys. 53, 3643 (1982).

[3] X. Boddaert, D. Stievenard, J.C. Bourgoin, Phys. Rev. B 40, 1051 (1989). 